\title{
Evaluación de cambios en el nivel de depresión de usuarios de un programa de bienestar
}

\author{
Evaluating changes in a welfare programme users' levels of \\ depression
}

Marisol Valencia-Cárdenas, Juan C. Salazar y Juan C. Correa-Morales

Universidad Nacional de Colombia, Sede Medellín. Medellín, Colombia. mvalencia@unal.edu.co, marisol.valencia@correo.upb.edu.co,jcsalaza@unal.edu.co,jccorrea@unal.edu.co

Recibido 15 Noviembre 2010/Enviado para Modificación 15 Julio 2011/Aceptado 10 Agosto 2011

\section{RESUMEN}

Objetivo La evaluación de impacto de un programa comunitario algunas veces se basa en técnicas estadísticas como modelos de regresión lineal, y tiene como objetivo cuantificar su efecto sobre aspectos sociales o el bienestar general de la comunidad. No es frecuente utilizar técnicas estadísticas con estructura de correlación entre los sujetos evaluados que permitan modelar variables de tipo social, o sicológica. Esta estrategia de análisis incorpora más información y por lo tanto debe producir resultados más precisos.

Métodos En este trabajo se muestra una evaluación de impacto de los efectos producidos sobre el nivel de depresión de una población participante de un grupo de acciones del INDER Medellín en el año 2005, usando modelos lineales mixtos. Resultados Se identificaron variables importantes que reflejan resultados de impacto positivos, como el tiempo de intervención y la edad.

Conclusiones Participar en las actividades de bienestar del INDER contribuye a disminuir los niveles de depresión.

Palabras Clave: Modelos lineales, técnicas de evaluación, salud mental, depresión (fuente: DeCS, BIREME).

\section{ABSTRACT}

Objective Statistical techniques such as linear regression models are sometimes used for evaluating a community programme's impact, especially when the goal is to quantify its effects on social aspects or a particular community's overall welfare. Statistical techniques taking intra-subject correlation into account, thereby allowing social or psychological variables to be modelled, are not normally used. Such modelling strategy takes more information into account and must thus yield more accurate results than those based solely on linear regression models. 
Methods This paper evaluates (by means of a linear mixed model) the impact of an Institute of Sport and Recreation (Instituto de Deportes y Recreación - INDER) programme run in 2005 in Medellín on a participating population's levels of depression.

Results Important variables such us intervention time and age were identified as important predictors which led to reducing depression.

Conclusions Participating in INDER's welfare programme contributed towards reducing levels of depression.

Key Words: Linear model, evaluation technique, mental health, depression (source: $\mathrm{MeSH}, \mathrm{NLM}$ ).

a evaluación de impacto de un programa comunitario se ha implementado como una técnica en busca de alternativas que midan la efectividad de políticas o programas (1). Las políticas públicas formuladas por entes estatales, se diseñan como lineamientos sobre poblaciones, obedeciendo a sus necesidades (2), sobre las que diseñan programas de intervención para el logro de metas comunitarias, desarrollo o mejoramiento de calidad o condiciones de vida y se establecen sistemas de seguimiento y control a través de monitoreos y posteriores evaluaciones, como la Evaluación de Impacto Social, que comprende los procesos de análisis, seguimiento y gestión de las consecuencias sociales voluntarias e involuntarias, tanto positivas como negativas de las intervenciones (1).

Las aproximaciones sobre la intervención de actividad física y deportiva se han dado a partir de diferentes investigaciones epistemológicas. Una de estas es el trabajo de Correa y González (2003), en el cual plantean que la familia representa condiciones no propicias para una adecuada socialización de sus miembros por evidencia de problemas de violencia, madre-solterismo, delincuencia, entre otros aspectos, mientras que la actividad recreativa y deportiva dada por diferentes medios educativos, incide positivamente en la dinámica de las relaciones sociales (3). El Instituto de Deportes y Recreación de Medellín, INDER, promueve esta dinámica por medio de las diversas actividades que ofrece de forma gratuita, y establece algunas metodologías para la evaluación de su efectividad.

Dada la hipótesis sobre la situación sin la intervención, una respuesta se aproxima con un modelo contrafactual: describir la situación hipotética sobre el bienestar en ausencia del programa, es decir, ¿Cuáles serían las condiciones, respuestas o resultados sin que el programa hubiera tenido lugar en la población o comunidad? Tal caso es utilizado por el programa de Familias en Acción, 
con presupuesto del Banco Mundial y en el que se evalúa el impacto a partir de información recolectada de un grupo tratamiento (intervenido) y un grupo control (no intervenido), de manera que se generen indicadores comparables (4). Por ello, la medición de una respuesta antes de una intervención asume el estado de ausencia del programa, para ser comparada con una respuesta posterior a su implementación, el cual es el propósito en este trabajo. La hipótesis de la evaluación de impacto tiene un punto de partida fundamental: ¿Cuál hubiera sido la situación si la intervención no hubiera tenido lugar? $(5,6)$.

Por medio de este trabajo se propone una alternativa de análisis estadístico para evaluar el efecto de un grupo de acciones del INDER Medellín, en el nivel de depresión de un grupo de personas adscritas en un periodo de 6 meses. Este análisis se basa en la implementación de un modelo lineal mixto con medidas repetidas, usando unos datos recolectados por dicho instituto. Estos datos se refieren a participantes de las acciones del proyecto denominado Estilos de Vida Saludable, año 2005, a quienes se les aplicó el Test de Depresión de Zung (7), y se les registraron otras características demográficas de interés.

\section{MÉTODOS}

En esta sección se describen los conceptos y metodologías utilizadas para el desarrollo de este trabajo, que parten desde el muestreo realizado, y la definición del modelo lineal mixto ajustado.

Descripción del muestreo

Se citaron adultos entre 18 y 45 años de edad de los estratos 1,2 y 3 de Medellín estratificando por comuna y se logró recolectar un total de 1023 personas en la primera etapa, predominando personas del género femenino (8). Sin embargo, en la segunda etapa llegaron 319 de estos 1023, que fue el total utilizado en este trabajo. Es decir, se utilizó solamente la información completa de personas antes y después de la intervención.

Estas personas llegaron a puntos de atención por zona y un grupo de profesionales se encargó de realizarles pruebas médicas, de relaciones sociales y sicológicas, dentro de las cuales se encuentra la medición del nivel de depresión usando el test de Zung, permitiendo la comparación antes y después a los mismos individuos. 
Test de Depresión de Zung

La Escala Autoaplicada de Depresión de Zung (Self-Rating Depression Scale, SDS), fue desarrollada por Zung en 1965. Es una escala de cuantificación de síntomas de base empírica y derivada en cierto modo de la escala de depresión de Hamilton, que da mayor peso al componente somático-conductual del trastorno depresivo. Lainformación, descripción, la bibliografía de algunos trabajos realizados sobre el tema, y el test se encuentran disponibles en internet (9). Es de anotar que este test está validado en nuestro medio y es de amplio uso (7).

El puntaje de Depresión se obtiene a partir de valoraciones de las respuestas de cada una de sus 20 preguntas, y se presentan las siguientes escalas: Normal de 0-49.9, Leve: 50-59.9, Moderada: 60-69.9, Extrema: 70 o más.

El test realizado consta de 20 preguntas, algunas de las cuales son:

1. ¿Se siente triste y decaído(a)?

2. ¿Se siente mejor en las mañanas?

3. ¿Tiene usted ganas de llorar frecuentemente?

10. ¿Se siente usted cansado(a) sin razón aparente?

Según la respuesta dada en una de estas categorías: Nunca o muy pocas veces, Algunas veces, Muchas veces, y casi siempre o siempre, se asigna una calificación de la cual se obtiene un primer puntaje que corresponde al $80 \%$ del total, por tanto, se establece una regla de 3, a partir de la cual, se clasifica a la persona en una de las siguientes categorías:

Descripción de la Población

La información de los participantes incluye su estrato socioeconómico, edad, zona, comuna, y el número de grupos con los que la persona interactúa. Un resumen de las estadísticas para estas variables y otras relacionadas con mediciones de comportamiento, se consignan en la Tabla 1.

Población

Base de datos: Se registró información de 319 individuos participantes de la acción Estilos de Vida Saludable del INDER en el año 2005. La muestra aleatoria se tomó de una base recolectada con un muestreo aleatorio estratificado en todas las comunas de la ciudad de Medellín, donde el programa venía realizando la intervención.

Variable respuesta de interés: Depresión, obtenido con el Test de Depresión de Zung (7). Validado en la versión original, el alfa de Cronbach fue 0.875, la 
sensibilidad 0.96 , la especificidad 0.71 , la kappa 0.441 y el área bajo de la curva ROC 0.86 para un punto de corte de 53 (idem).

Técnica de evaluación de impacto: Modelos lineales mixtos con respuesta repetida correlacionada (10-14).

Tabla 1. Datos sobre los usuarios participantes (Total: 319)

\begin{tabular}{|c|c|}
\hline Edad & $\begin{array}{c}\text { Media } \pm \text { DE: } 33 \pm 8 \\
\text { Mediana }=34 \\
\text { Percentiles } 25 \% \text { y } 75 \%= \\
28 \text { y } 40\end{array}$ \\
\hline & Frecuencias (\%) \\
\hline \multicolumn{2}{|l|}{ Género } \\
\hline Mujeres & $296(93)$ \\
\hline Hombres & $23(7)$ \\
\hline \multicolumn{2}{|l|}{ Zona } \\
\hline 1 & $88(28)$ \\
\hline 2 & $63(20)$ \\
\hline 3 & 47 (15) \\
\hline 4 & $14(4)$ \\
\hline 6 & 36 (11) \\
\hline \multicolumn{2}{|l|}{ Corregimientos } \\
\hline Palmitas (50) & $8(3)$ \\
\hline San Cristóbal (60) & $10(3)$ \\
\hline Altavista (70) & $13(4)$ \\
\hline San Antonio de Prado (80) & $26(8)$ \\
\hline Santa Elena (90) & $14(4)$ \\
\hline \multicolumn{2}{|l|}{ Estrato } \\
\hline 1 & $41(13)$ \\
\hline 2 & $200(63)$ \\
\hline 3 & $76(24)$ \\
\hline 4 & $2(1)$ \\
\hline \multicolumn{2}{|c|}{ Número de grupos con los que la persona interactúa } \\
\hline 0 & $2(0,6)$ \\
\hline 3 & $1(0,2)$ \\
\hline 4 & $16(5)$ \\
\hline 5 & 77 (24) \\
\hline 6 & $79(25)$ \\
\hline 7 & $73(23)$ \\
\hline 8 & $30(9)$ \\
\hline 9 & $21(7)$ \\
\hline$\geq 10$ & $20(6.3)$ \\
\hline
\end{tabular}

A continuación se describe brevemente el modelo lineal mixto empleado para la estimación de dicho impacto sobre la respuesta nivel de depresión de Zung (7).

Modelo lineal mixto para el análisis de impacto

Los modelos lineales mixtos son apropiados para determinar efectos sobre variables de respuesta cuando las estructuras de correlación entre individuos se deben tener en cuenta. Esta característica facilita la interpretación y análisis de 
asociaciones importantes con las que pueden delinearse planes de acción o de mejoramiento. Por el contrario, estas estructuras no se pueden incorporar de manera explícita en los modelos de regresión lineal clásicos ocasionando una pérdida de información importante.

Los modelos lineales mixtos se pueden usar cuando existen datos que tienen una estructura de correlación. Por ejemplo, el peso tomado como medida repetida en diferentes tiempos a un mismo individuo, donde la correlación se da entre medidas de un mismo sujeto o unidad experimental. Para estudiar más a fondo la forma de este modelo se puede consultar Lange y Ryan (10) o McCulloch y Searle (12). Este modelo se puede adaptar también para analizar respuestas categóricas de tipo ordinal o nominal.

En este documento se considera sólo el modelo denominado Modelo lineal Mixto generalizado, con una respuesta dicótoma, categorizada como: 1: la persona tiene depresión, 0: no tiene depresión.

\section{RESULTADOS}

En esta sección se presentan los resultados preliminares encontrados, y posteriormente, los efectos detectados con la técnica estadística utilizada para la estimación respectiva.

Tabla 2. Cantidad de usuarios por escala de clasificación

\begin{tabular}{lccccc}
\multicolumn{3}{c}{ de la depresión } \\
\hline \multirow{2}{*}{ Nivel de depresión } & \multicolumn{2}{c}{ Antes } & \multicolumn{2}{c}{ Después } \\
& & No. & $\%$ & No. & $\%$ \\
\hline Normal & $(0,49.9)$ & 214 & 67,1 & 232 & 72.73 \\
Leve & $(49.9,59.9)$ & 56 & 17,6 & 54 & 16.93 \\
Marcada & $(59.9,69.9)$ & 31 & 9.7 & 20 & 6.27 \\
Extrema & $(69.9,100)$ & 18 & 5.6 & 13 & 4.08 \\
\hline
\end{tabular}

En la Tabla 2 se observa que antes de la intervención había más personas con depresión que en estado normal.

Lo anterior permite visualizar de manera exploratoria, cómo la depresión parece tener menores valores después del tiempo de intervención. Pero es interesante ver qué asociación tiene la depresión con otras de las variables consideradas.

A continuación se muestran los resultados obtenidos al estimar el modelo lineal mixto generalizado con la variable depresión categorizada como: 1: la persona tiene depresión, 0: no tiene depresión. 
Ajuste del modelo lineal mixto generalizado

Es posible usar el programa R (15) para estimar el modelo lineal mixto generalizado, utilizando la variable respuesta dicótoma para analizar el efecto de las siguientes covariables sobre la probabilidad de tener depresión:

TIEMPO: tiempo de intervención de las actividades, con valores 0: antes de la intervención, 1: seis meses después de la intervención.

NUMGRUP, número de grupos con los que la persona socializa.

El puntaje de depresión fue cambiado por la variable dicótoma como se ve en la Tabla 3.

Tabla 3. Variable dicótoma de la depresión

\begin{tabular}{lc}
\hline \multicolumn{1}{c}{ Nivel de Depresión } & Dicótoma \\
\hline Normal (0-49.9) & 0 \\
Leve (50-59.9) & 1 \\
Moderada (60-69.9) & 1 \\
Extrema (70 o más) & 1 \\
\hline
\end{tabular}

Así, los niveles Leve a Extrema corresponden a tener depresión. El modelo permitirá estimar la probabilidad de tener depresión en presencia del tiempo de intervención y el número de grupos.

Para estimar el modelo se utilizó la función glmmPQL, del paquete MASS del programa $\mathrm{R}$ (15). A partir de este se observaron los resultados vistos en la Tabla 4.

Tabla 4. Modelo mixto para la medición de la probabilidad de tener depresión

\begin{tabular}{ccccc}
\hline Efecto & Estimador ${ }^{\mathrm{a}}$ & Valor $\mathrm{p}^{\mathrm{b}}$ & \multicolumn{2}{c}{$\mathrm{Cl}$ al $90 \%{ }^{\mathrm{c}}$} \\
\hline TIEMPO & -0.283 & 0.08 & -0.553 & -0.013 \\
NUMGRUP & -0.152 & 0.02 & -0.261 & -0.043 \\
\hline a Parámetros $\beta_{\mathrm{i}}$ del modelo estimado; ${ }^{\mathrm{b}}$ Valor $\mathrm{p}$ para probar la hipótesis de que $\beta_{\mathrm{i}}=0$ vs $\beta_{\mathrm{i}}$ es \\
diferente de $0 ;{ }^{c}$ Intervalo de confianza al $90 \%$ para los parámetros $\beta_{\mathrm{i}}$, con la distribución $\mathrm{t}$
\end{tabular}

El modelo refleja que tanto el tiempo de permanencia en las actividades, como el aumento del número de grupos de socialización generan una disminución de la probabilidad de tener depresión, lo cual se refleja en los valores negativos de los intervalos al $90 \%$ de confianza para los efectos de cada variable, mostrando un impacto beneficioso para el conjunto de personas participantes de las actividades del programa.

Este modelo estimado sin restringirse al supuesto de normalidad, refleja el impacto beneficioso producido por la intervención del programa, ya que se 
ve que los efectos del tiempo, el número de grupos, hacen que la probabilidad de tener depresión sea menor.

\section{DISCUSIÓN}

A través de la técnica empleada con el modelo lineal mixto generalizado, fue posible detectar impactos positivos en la reducción de la depresión de un grupo de personas a partir del trabajo desarrollado por el grupo de acciones ofertadas a través del Instituto de Deportes y Recreación de Medellín, INDER. Este impacto encontrado, permite verificar que estas actividades generan actitudes positivas en el mejoramiento del nivel de depresión de un conjunto de personas de 18 a 45 años de la comunidad participante de las mismas. Este modelo tiene en cuenta la asociación y la variabilidad intrasujeto por lo que incorporan más información que en los modelos de regresión múltiple.

Evidenciando estos impactos, se aprecia en el modelo estimado una disminución de la depresión después de un tiempo de 6 meses de intervención de la actividad, y una disminución a medida que la persona se relaciona socialmente con más grupos, agregando que la probabilidad de tener depresión disminuye en estos escenarios. El factor de socialización que también se promueve con las actividades del programa, es otro beneficio importante que muestra cómo incide en la disminución de la depresión •

Agradecimientos: Los autores agradecen al INDER Medellín por proporcionar la información de sus bases de datos para la aplicación de esta metodología, así como su autorización para divulgar el resultado de este estudio. A la Facultad de Ciencias de la Universidad Nacional, Sede Medellín. Trabajo patrocinado con recursos del proyecto "DIME 20101007954, Convocatoria Nacional 2009, Universidad Nacional de Colombia, Sede Medellín".

\section{REFERENCIAS}

1. Vanclay F. Principios internacionales de la evaluación de impacto social. Serie de publicaciones oficiales No. 2; 2003.

2. Rico C. La generación de Capital Social a partir de actividades deportivas y recreativas como alternativa para promover el desarrollo integral del país. INDER Medellín; 2006.

3. Correa E, González C. Las expresiones motrices como alternativa para la reconstrucción de tejido social. Experiencia evaluativa de la intervención social en la Escuela de Iniciación y Formación deportiva Santo Domingo Savio. Revista Educación Física y Deporte. 2003 Enero-octubre; 22(1): 143-147.

4. SINERGIA. Primer seguimiento evaluación de impacto del Programa Familias en Acción. Departamento Nacional de Planeación; Disponible en: http://www.dnp.gov.co/ portalweb/Programas/Sinergia/HerramientasyProductosdelSistema/ Basesdedatos.aspx. Consultado en: Agosto de 2011. 
5. Ravillion M. The Mystery of the Vanishing Benefits: Ms. Speedy Analyst's Introduction to Evaluation. Policy Research Working Paper 2153. The World Bank Development Research Group, July; 1999.

6. Ravillion M. Evaluating Anti-Poverty Programs. World Bank Policy Research Working Paper 3625. June; 2005.

7. Díaz LA, Campo A, Rueda GE, Barros JA. Propuesta de una versión abreviada de la escala de Zung para depresión. Colombia Médic. 2005; 36(3): 168-172.

8. Valencia M, Gómez LA, Arango C, Gutiérrez A, Sora E, Vélez D et al. Efectos de la actividad física con la intervención de las actividades del proyecto Estilos de Vida Saludable en el año 2005. Oficina de planeación INDER, Medellín; 2006.

9. Rozados.¿ ¿Tiene usted depresión? [Internet]. Disponible en: http://www.depresion.psicomag.com/ esc_zung.php Consultado: Abril de 2010.

10. Lange N, Ryan L. Assessing normality in random effects models. Ann. Statist. 1989: 17: 624-642.

11. Laird NM, Ware JH. Random effects models for longitudinal data. Biometrics. 1982; 38: 963-974.

12. McCulloch CE, Searle SR. Generalized, Linear and Mixed Models. Wiley Series in Probability and Statistics; 2000. p.156.

13. Verbeke G, Molenberghs G. Linear Mixed Models for Longitudinal Data. New York: Springer; 2001.

14. West BT, Welch KB, Galecki AT. Linear Mixed Models. A practical Guide Using Statistical Software. University of Michigan, USA. Chapman \& Hall; 2007.

15. The R project for Statistical Computing. The R Foundation for Statistical Computing. [Internet]. Disponible en: www.cran.r-project.org Consultado: Abril de 2010. 